\title{
Capacitance, Charge Fluctuations and Dephasing In Coulomb Coupled Conductors
}

\author{
Markus Büttiker \\ Université de Genève, Département de Physique Théorique, \\ CH-1211 Genève 4, Switzerland
}

\begin{abstract}
The charge fluctuations of two nearby mesoscopic conductors coupled only via the long range Coulomb force are discussed and used to find the dephasing rate which one conductor exerts on the other. The discussion is based on a formulation of the scattering approach for charge densities and the density response to a fluctuating potential. Coupling to the Poisson equation results in an electrically self-consistent description of charge fluctuations. At equilibrium the low-frequency noise power can be expressed with the help of a charge relaxation resistance (which together with the capacitance determines the RC-time of the structure). In the presence of transport the low frequency charge noise power is determined by a resistance which reflects the presence of shot noise. We use these results to derive expressions for the dephasing rates of Coulomb coupled conductors and to find a self-consistent expression for the measurement time.
\end{abstract}

\section{Introduction}

Investigations of time-dependent current fluctuations of mesoscopic systems have been widely used to obtain information which cannot be extracted from conductance measurements alone [1]. In this work we are interested in the fluctuations of the charge in a volume element inside the electrical conductor. If the volume element is made very small the fluctuations of interest are thus the fluctuations of the local electron density. Such fluctuations can be detected, for instance by measuring the current induced into a nearby gate [2] or a small cavity as shown in Fig. 1. Through the long range Coulomb interaction a charge fluctuation above the average equilibrium value of the charge in the conductor generates additional electric fields which lead to a charge reduction at the surface of the gate. The reduction of charge at the gate surface is accomplished by a flow of carriers out of the contact of the gate [2]. The conductor can be in an equilibrium state in which case the charge fluctuations are associated with Nyquist noise or it can be in a transport state and the charge fluctuations are those that are generated by shot noise.

Charge fluctuations can be detected not only by direct capacitive probing. In an experiment by Buks et al. [3] charge fluctuations are observed through conductance measurements: the charge fluctuations of two conductors in close proximity can give rise to an additional dephasing rate which a carrier in one conductor experiences due to the presence of the other conductor. In the experiment of Buks et al. [3] an Aharonov-Bohm ring with a quantum dot in one of its 
arms is in close proximity to another conductor which forms a QPC (quantum point contact). The presence of the QPC leads to a reduction of the AharonovBohm interference oscillations. In the experiment of Sprinzak et al. [4 a double quantum dot is brought into the proximity of a conductor in a high magnetic field and the broadening of the Coulomb blockade peak due the charge fluctuations in the edge states of the nearby conductor are measured. Theoretical discussions

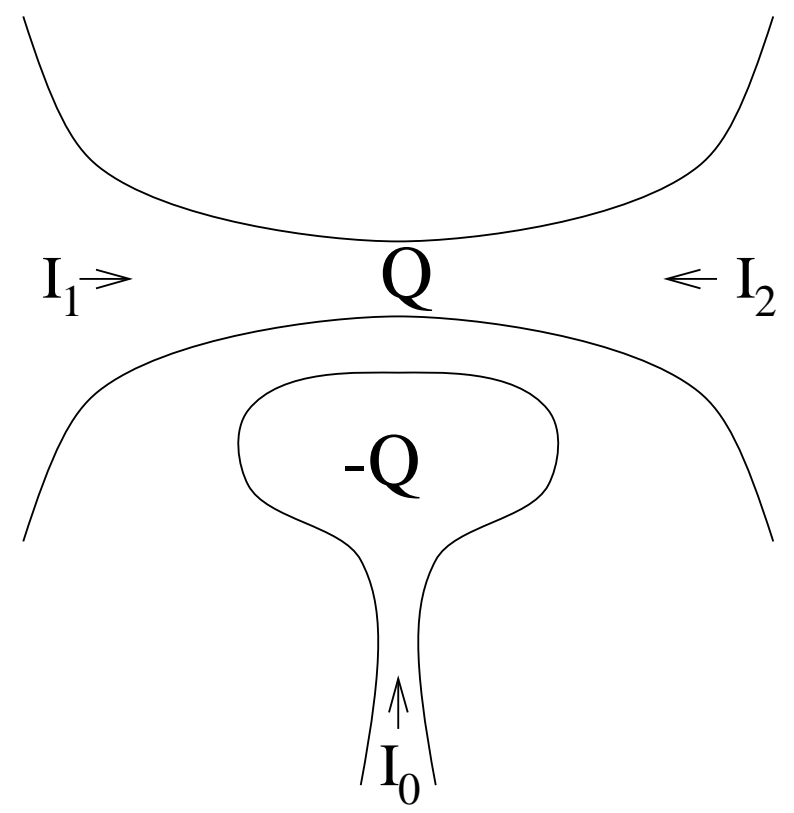

Fig. 1. Cavity with a charge deficit $-Q$ in the proximity of a quantum point contact with an excess charge $Q$. The dipolar nature of the charge distribution ensures the conservation of currents $I_{0}, I_{1}$ and $I_{2}$ flowing into this structure. After [2].

of dephasing rates in coupled systems are given in Refs. [5, 6, 7, 8, 9, 10,11]. Harris and Stodolsky [5] 9] view this as a quantum measurement problem in which the state of one system is measured with the help of another. In this work, as well as in the experimental work of Buks et al. [3] the time it takes to ascertain the state of the measured system, the measurement time, is identified with the dephasing time. Gurvitz investigates the time-evolution of the density matrix of the system that is measured [6]. Aleiner et al. [7] relate the dephasing rate to the orthogonality catastrophe which occurs if an additional carrier is added to the ground state of the system. Levinson derives a dephasing rate in terms of the charge fluctuation spectrum of non-interacting carriers [8]. The approach which we discuss here also relates the dephasing rate to the charge fluctuation spectrum. However, in contrast to the discussions presented in Refs. [5, 6, 8, 9] we emphasize an electrically self-consistent approach which takes into account that charging 
even in open conductors such as QPC can be energetically expensive 10. This approach is applicable to a wide range of geometries and in Refs. 10.11] has been used to present a self-consistent treatment of charge fluctuations in edge states. Our discussion can be compared with Ref. [12] which treats fluctuations as a free electron problem.

It is interesting to notice that in discussions of charge fluctuations in systems that are composed entirely of components in which charge quantization is important, theoretical work [13, 14, 15, 16, 17] carefully discusses the various capacitance coefficients which determine the charging energies of the system and the coupling. On the other hand, when subsystems like QPC's are discussed, electric carriers are treated as if they were non-interacting entities [14, 15, 16, 17. Clearly, a QPC has also a capacitance. A QPC can be characterized by a capacitance which describes its self-polarization [18, 19,20]. This self-polarization corresponds to charge accumulation on one side of the quantum point contact and charge depletion on the other side of the quantum point contact 18,19]. The self-polarization does not change the overal charge of the QPC and is thus here not of primary importance. But a quantum point contact can be charged vis-avis the gates 21] or vis-a-vis any other conductor. This leads to a net charge on the quantum point contact and is the dominant process by which the quantum point contact interacts with an other conductor 21].

Therefore, like in the systems which exhibit Coulomb blockade, we can ask: "What is the dependence of a charge fluctuation spectrum of a QPC on its capacitance?", or "How does the additional dephasing rate generated by the proximity of a QPC depend on the capacitance of the quantum point contact?" If the relevant capacitance were a mere geometrical quantity these questions might just determine some prefactors left open in previous work. However, the charge screening of a QPC is non-trivial since the density of states of a quantum point contact [2] (at least in the semi-classical limit) diverges for a gate voltage at which a new channel is opened (quantum tunneling limits the density of states).

The aim of this work is to present a simple self-consistent discussion of charge fluctuation spectra of Coulomb coupled conductors and to use these spectra to find dephasing rates and self-consistent expression for the measurement time. We assume that the ground state of the system has been determined and investigate small deviations away from the ground state. The approach which we present combines the scattering approach with the Poisson equation and treats interactions in the random phase approximation [10]. This approach has been used with some success to treat the dynamic conductance 22,23. of mesoscopic systems, their non-linear I-V-characteristics [24,25,26, and higher harmonics generation [25. Indeed there is a close connection between the charge fluctuation spectra which we obtain and the dynamic conductances of a mesoscopic system 22,21. We show that the charge noise power of the equilibrium charge fluctuations can at low frequencies be characterized by a charge relaxation resistance $R_{q}$. Together with the capacitance this resistance determines the $R C$-time of the mesoscopic structure 222]. Indeed, independent measurements of the capacitance and the resistance $R_{q}$, when compared with the results from a measurement of the de- 
phasing rate would provide an important overall test of the consistency of the theory. In the presence of transport, we deal in the low temperature limit with shot noise. In this case, the low frequency charge noise power is proportional to the applied voltage and proportional to a resistance $R_{v}$.

Some works advocate a perturbative treatment of coupled systems and present results which are proportional to the square of the coupling constant. In contrast, the self-consistent approach discussed here leads to a more intersting dependence on the bare coupling constant 10] (in the physically most relevant case we actually find a dephasing rate which is proportional to one over the square of the coupling strength).

In this work, we treat only the symmetric QPC Coulomb coupled to another system. This limitation is motivated by the fact that the QPC is the most widely investigated example. This should permit a most direct comparison of the selfconsistent approach advocated here with the results in the literature. For a review which addresses a wider range of geometries we refer the reader to [27]. We also restrict ourselves to the case where linear screening is applicable.

An evaluation of the dephasing rates requires a discussion of the potential fluctuations (or equivalently, the charge fluctuations). We will need only the zerofrequency limit of the potential fluctuation spectrum, and it is thus sufficient to find the zero-frequency, white noise limit of the charge fluctuations.

\section{The effective interaction}

To be definite, consider the conductors of Fig. 1. We first investigate the relation between voltage and charges of these two conductors for small deviations of the applied voltages away from their equilibrium value. We assume that each electric field line emanating from the cavity ends up either again on the cavity or on the QPC. There exists a Gauss volume which encloses both conductors which can be chosen large enough so that the electrical flux through its surface vanishes 23. Consequently, the charge on the two conductors is conserved. Any accumulation of charge at one location within our Gauss volume is compensated by a charge depletion at another location within the Gauss volume. The variation of the charge brought about by a small change of the applied voltage is thus of a dipolar nature.

To keep the discussion simple, we consider here the case that each conductor is described by a single potential only. The charge and potential on the cavity are denoted by $d Q_{0}$ and $d U_{0}$ and on the QPC by $d Q_{1}$ and $d U_{1}$. A more accurate description can be obtained by subdividing the conductor into a number of volume elements [21] or in fact by using a continuum description [23]. The essential elements of our discussion do, however, already become apparent in the simple case that each conductor is described only by one potential and we will treat only this limiting case in this work. The Coulomb interaction is described with the help of a single geometrical capacitance $C$. The charges and potentials of the two conductors are then related by

$$
d Q_{0}=C\left(d U_{0}-d U_{1}\right)
$$




$$
d Q_{1}=C\left(d U_{1}-d U_{0}\right) .
$$

The two equations can be thought off as a discretized version of the Poisson equation. Note that according to Eqs. (1, 22) we have $d Q_{0}+d Q_{1}=0$.

We now complement these two equations by writing the charges $d Q_{i}$ as a sum of an external (or bare) charge calculated for fixed internal potentials $U_{0}$ and $U_{1}$ and an induced charge generated by the response of the potential due to the injected charges. The additional charge injected into the cavity (which we denote by $e d N_{0}$ ) due to an increase of the reservoir voltage by $e d V_{0}$ is $e d N_{0}=e D_{0} e d V_{0}$. Here $D_{0}$ is the total density of states at the Fermi energy of the cavity of the region in which $d U_{0}$ deviates from its equilibrium value. The induced charge is $-e^{2} D_{0} d U_{0}$. It is negative since the Coulomb interaction counteracts charging. It is also determined by the total density of states since the integrated Lindhard function is given by the density of states [28. Thus the charge on the cavity is

$$
d Q_{0}=\left(e d N_{0}-e^{2} D_{0} d U_{0}\right)
$$

To find the charge on the QPC we must take into account that there are two reservoir potentials which we denote by $d V_{1}$ and $d V_{2}$. An increase in the potential of contact of reservoir 1 (at constant internal potential $U_{1}$ ) does not fill all the available states but only a portion. The density of states [21] of carriers incident from reservoir 1 is denoted by $D_{11}$ and is called the injectance of contact 1 . Thus the charge injected at constant internal potential by an increase of the voltage $V_{1}$ is $e D_{11} e d V_{1}$. Similarly an increase of the potential at reservoir 2 leads to an additional charge $e D_{12} e d V_{2}$. Here $D_{12}$ is the injectance of reservoir 2. Together these two (partial) density of states are equal to the total density of states of the quantum point contact $D_{11}+D_{12}=D_{1}$. Thus the total injected charge on conductor 1 is $e d N_{1}=e D_{11} e d V_{1}+e D_{12} e d V_{2}$. Screening is against the total density of states and thus a variation of the internal potential $d U_{1}$ generates an induced charge given by $e^{2} D_{1} d U_{1}$. The charge $d Q_{1}$ is the sum of these three contributions,

$$
d Q_{1}=\left(e d N_{1}-e^{2} D_{1} d U_{1}\right) .
$$

We arrive thus at the following self-consistent equations relating charges and potentials,

$$
\begin{aligned}
& d Q_{0}=C\left(d U_{0}-d U_{1}\right)=\left(e d N_{0}-e^{2} D_{0} d U_{0}\right), \\
& d Q_{1}=C\left(d U_{1}-d U_{0}\right)=\left(e d N_{1}-e^{2} D_{1} d U_{1}\right) .
\end{aligned}
$$

We can use these equations to express the internal potentials $U_{i}$ in terms of the injected charges $e d N_{i}$. We find $d U_{i}=e \sum_{j} G_{i j} d N_{j}$ with an effective interaction $G_{i j}$ given by

$$
\mathbf{G}=\frac{C_{\mu}}{e^{2} D_{0} e^{2} D_{1} C}\left(\begin{array}{l}
C+e^{2} D_{1} C \\
C
\end{array} \begin{array}{l}
C+e^{2} D_{0}
\end{array}\right) .
$$

Here $C_{\mu}$ is the electrochemical capacitance

$$
C_{\mu}^{-1}=C^{-1}+\left(e^{2} D_{0}\right)^{-1}+\left(e^{2} D_{1}\right)^{-1}
$$


which is the series capacitance of the geometrical contribution $C$ and the density of states of the two conductors 22]. Note that in contrast to perturbation treatments, the effective coupling element $G_{12}$ is not proportional to $e^{2} / C$ but in general is a complicated function of this energy. We will use the effective interaction in Section 7 to find the measurement time. First, however, we will now use the effective interaction to express the true charge fluctuations in terms of the bare fluctuations.

We are interested not in the average quantities discussed above but in their dynamic fluctuations. To this extend we now re-write Eqs. (5) and (6) for the fluctuating quantities. In a second quantization approach the fluctuating quantities are described with the help of operators, $\hat{Q}_{i}$ for the true charges, and the potentials $\hat{U}_{i}$ on the two conductors, $i=0,1$. As for the average charge, the fluctuating charge can also be written in terms of bare charge fluctuations $e \hat{\mathcal{N}}_{i}$ (calculated by neglecting the Coulomb interaction) counteracted by a screening charge $e D_{i} e \hat{U}_{i}$. Below, we will give explicit expressions for all these operators. Instead of Eqs. (5) and (6) we now have,

$$
\begin{aligned}
& \hat{Q}_{0}=C\left(\hat{U}_{0}-\hat{U}_{1}\right)=e \hat{\mathcal{N}}_{0}-e^{2} D_{0} \hat{U}_{0}, \\
& \hat{Q}_{1}=C\left(\hat{U}_{1}-\hat{U}_{0}\right)=e \hat{\mathcal{N}}_{1}-e^{2} D_{1} \hat{U}_{1} .
\end{aligned}
$$

Clearly, if we consider simply the average of these equations, they must reduce to Eq. (5) and (6). The fluctuations are determined by the off-diagonal elements of the charge and potential operators. Below we will specify these expressions in detail. Solving these equations for the potential operators, we find $\hat{U}_{i}=e \sum_{j} G_{i j} \hat{\mathcal{N}}_{j}$ with the effective interaction $G_{i j}$ given by Eq. (7).

Let us now introduce the noise power spectra of the bare charges, $S_{N_{i} N_{i}}(\omega)$ for each of the conductors. The spectrum of the bare charge fluctuations is defined as

$$
S_{N_{i} N_{i}}(\omega) 2 \pi \delta\left(\omega+\omega^{\prime}\right)=\left\langle\hat{\mathcal{N}}_{j}(\omega) \hat{\mathcal{N}}_{j}\left(\omega^{\prime}\right)+\hat{\mathcal{N}}_{j}\left(\omega^{\prime}\right) \hat{\mathcal{N}}_{j}(\omega)\right\rangle
$$

with $\hat{\mathcal{N}}_{j}(\omega)=\hat{\mathcal{N}}_{j}(\omega)-\left\langle\hat{\mathcal{N}}_{j}(\omega)\right\rangle$, where $\hat{\mathcal{N}}_{j}(\omega)$ is the Fourier transform of the charge operator of conductor $j$.

The bare charge fluctuation spectra on different conductors are uncorrelated, $S_{N_{i} N_{j}}(\omega)=0$ for $i \neq j$. With the help of the effective interaction matrix, we can now relate the potential fluctuation spectra to the fluctuation spectra of the bare charges. In the zero-frequency limit we find,

$$
S_{U_{i} U_{j}}(0)=e^{2} \sum_{k} G_{i k} G_{j k} S_{N_{k} N_{k}}(0)
$$

Even though the bare charge fluctuations are uncorrelated, the potential fluctuations and the true charge fluctuations on the two conductors are correlated.

\section{Charge Relaxation Resistances}

It is useful to characterize the noise power of the charge fluctuations with the help of resistances. Consider first the case where both conductors are at equilibrium. 
Charge fluctuations on the conductors arise due to the random thermal injection of carriers. The bare charge fluctuation spectrum, normalized by the density of states $D_{i}$ of conductor $i$ has the dimension of a resistance. We introduce the charge relaxation resistance $R_{q}^{(j)}$ of conductor $j$,

$$
2 k T R_{q}^{(j)} \equiv e^{2} S_{N_{j} N_{j}}(0) /\left(e^{2} D_{j}\right)^{2}
$$

The charge relaxation resistance has a physical significance in a number of problems. In simple cases, $R_{q}$ together with an appropriate capacitance determines the $R C$-time of the mesoscopic structure 22]. The charge relaxation resistance can thus alternatively be determined by investigating the poles of the conductance matrix 23,22. The dynamic conductance matrix $G_{\alpha \beta}(\omega) \equiv$ $d I_{\alpha}(\omega) / d V_{\beta}(\omega)$ of our mesoscopic structure (QPC and cavity) which relates the currents $d I_{\alpha}(\omega)$ at a frequency $\omega$ at contact $\alpha$ to the voltages $d V_{\beta}(\omega)$ applied at contact $\beta$ has at low frequencies a pole determined by $\omega_{R C}=-i C_{\mu}\left(R_{q}^{(1)}+R_{q}^{(2)}\right)$. Alternatively we could carry out a low frequency expansion of the element $G_{00}(\omega)$ (the element of the conductance matrix which gives the current at the contact of the cavity in response to an oscillating voltage applied to the cavity) to find 22,23] that $G_{00}(\omega)=-i C_{\mu} \omega+C_{\mu}^{2} R_{q} \omega^{2}+\ldots$ Thus $R_{q}$ plays a role in many problems. The charge relaxation resistance differs from the dc-resistance. For instance a ballistic one-channel quantum wire connecting two reservoirs and capacitively coupled to a gate has for spinless carriers a dc-resistance of $R=h / e^{2}$ and a charge relaxation resistance [31] of $R_{q}=h / 4 e^{2}$. The dc-resistance corresponds to the series addition of resistances along the conductance path, whereas an excess charge on the conductor relaxes via all possible conductance channels to the reservoirs and thus corresponds to the addition of resistances in parallel. This is nicely illustrated for a chaotic cavity [32] connected via contacts with $M_{1}$ and $M_{2}$ perfectly transmitting channels to reservoirs and capacitively coupled to a gate. Its ensemble averaged dc-resistance is $R=\left(h / e^{2}\right)\left(M_{1}^{-1}+M_{2}^{-1}\right)$, whereas its charge relaxation resistance is $R_{q}=\left(h / e^{2}\right)\left(M_{1}+M_{2}\right)^{-1}$. Thus the dc-resistance is governed by the smaller of the two contacts, whereas the charge relaxation resistance is determined by the larger contact.

If the conductor is driven out of equilibrium with the help of an applied voltage $|V| \equiv\left|V_{1}-V_{2}\right|$, the thermal noise described by Eq. (13) can be overpowered by shot noise. For $e|V| \gg k T$ the charge fluctuation spectrum becomes proportional to the applied voltage and defines a resistance $R_{v}^{(j)}$ via the relation,

$$
2 e|V| R_{v}^{(j)} \equiv e^{2} S_{N_{j} N_{j}}(0) /\left(e^{2} D_{j}\right)^{2} .
$$

The resistance $R_{v}$ is thus a measure of the noise power of the charge fluctuations associated with shot noise.

Eq. (13) and Eq. (14) describe the behavior of $e^{2} S_{N_{j} N_{j}}(0) /\left(e^{2} D_{j}\right)^{2}$ in the limits $k T \gg e|V|$ and $k T \ll e|V|$. For fixed temperature as a function of voltage $e^{2} S_{N_{j} N_{j}}(0) /\left(e^{2} D_{j}\right)^{2}$ exhibits a smooth crossover from the equilibrium result Eq. (13) to Eq. (14) valid in the presence of shot noise. For the structure shown in Fig. 1 it is only the QPC (conductor 1) which can be brought out of equilibrium. 
The cavity, connected to a single lead always exhibits only thermal fluctuations and its charge relaxation resistance is characterized by $R_{q}^{0}$ even if the QPC is subject to shot noise.

\section{Bare Charge Fluctuations and the Scattering Matrix}

Let us now determine the charge operator for the bare charges (non-interacting carriers). The operator for the total charge on a mesoscopic conductor can be found from the current operator and by integrating the continuity equation over the total volume of the conductor. This gives a relation between the charge in the volume and the particle currents entering the volume. We obtain for the density operator [2]

$$
\hat{\mathcal{N}}(\omega)=\hbar \sum_{\beta \gamma} \sum_{m n} \int d E \hat{a}_{\beta m}^{\dagger}(E) \mathcal{D}_{\beta \gamma m n}(E, E+\hbar \omega) \hat{a}_{\gamma n}(E+\hbar \omega),
$$

where $\hat{a}_{\beta m}^{\dagger}(E)$ (and $\left.\hat{a}_{\beta m}(E)\right)$ creates (annihilates) an incoming particle with energy $E$ in lead $\beta$ and channel $m$. The element $\mathcal{D}_{\beta \gamma m n}(E, E+\hbar \omega)$ is the nondiagonal density of states element generated by carriers incident simultaneously in contact $\beta$ in quantum channel $m$ and by carriers incident in contact $\gamma$ in channel $n$. In particular, in the zero-frequency limit, we find in matrix notation [2.10],

$$
\mathcal{D}_{\beta \gamma}(E)=\frac{1}{2 \pi i} \sum_{\alpha} \mathbf{s}_{\alpha \beta}^{\dagger}(E) \frac{d \mathbf{s}_{\alpha \gamma}(E)}{d E} .
$$

Expressions of this type are known from the discussion of quantum mechanical time delay [33]. The sum of the diagonal elements of this matrix is the density of states of the conductor

$$
D(E)=\sum_{\beta} \operatorname{Tr}\left[\mathcal{D}_{\beta \beta}(E)\right]=\frac{1}{2 \pi i} \sum_{\alpha, \beta} \operatorname{Tr}\left[\mathbf{s}_{\alpha \beta}^{\dagger}(E) \frac{d \mathbf{s}_{\alpha \beta}(E)}{d E}\right],
$$

where the trace is over the quantum channels. $\mathcal{D}_{\beta \beta} \equiv \mathcal{D}_{\beta}$ is the injectance of contact $\beta$. (We used this density of states in the discussion leading to Eq. (3)).

The charge fluctuations are determined by the off-diagonal elements of Eq. (15). Proceeding as for the case of current fluctuations [34, 35. 1] we find for the fluctuation spectrum of the total charge

$$
S_{N N}(0)=2 h \sum_{\gamma \delta} \int d E \operatorname{Tr}\left[\mathcal{D}_{\gamma \delta}^{\dagger} \mathcal{D}_{\delta \gamma}\right] f_{\gamma}(E)\left(1-f_{\delta}(E)\right) .
$$

The spectrum of the bare charge fluctuations has to be determined for each conductor separately using its scattering matrix. We now go on to find specific expression for this spectrum for the QPC. 


\section{Charge relaxation resistance of a quantum point contact}

To illustrate the preceding discussion, we now consider specifically the charge relaxation resistance and subsequently the resistance $R_{v}$ of a QPC. For simplicity, we consider a symmetric QPC (the asymmetric case [- 1 ] is treated in 10, 12]): For a symmetric scattering potential the scattering matrix (in a basis in which the transmission and reflection matrices are diagonal) is for the $n$-th channel of the form

$$
s_{n}(E)=\left(\begin{array}{ll}
-i \sqrt{R_{n}} \exp \left(i \phi_{n}\right) & \sqrt{T_{n}} \exp \left(i \phi_{n}\right) \\
\sqrt{T_{n}} \exp \left(i \phi_{n}\right) & -i \sqrt{R_{n}} \exp \left(i \phi_{n}\right)
\end{array}\right),
$$

where $T_{n}$ and $R_{n}=1-T_{n}$ are the transmission and reflection probabilities and $\phi_{n}$ is the phase accumulated by a carrier in the $n$-th eigen channel. We find for the elements of the density of states matrix, Eq. (16),

$$
\mathcal{D}_{11}=\mathcal{D}_{22}=\frac{1}{2 \pi} \frac{d \phi_{n}}{d E}, \mathcal{D}_{12}=\mathcal{D}_{21}=\frac{1}{4 \pi} \frac{1}{\sqrt{R_{n} T_{n}}} \frac{d T_{n}}{d E} .
$$

With these density of states matrix elements, we can determine the particle fluctuation spectrum, Eq. (18) in the white-noise limit, and $R_{q}$ with the help of Eq. (13)

$$
R_{q}=\frac{h}{4 e^{2}} \frac{\sum_{n}\left[\left(\frac{d \phi_{n}}{d E}\right)^{2}+\frac{1}{4 T_{n} R_{n}}\left(\frac{d T_{n}}{d E}\right)^{2}\right]}{\left[\sum_{n} \frac{d \phi_{n}}{d E}\right]^{2}} .
$$

Eq. (21) is still a formal result, applicable to any symmetric (two-terminal) conductor. To proceed we have to adopt a specific model for a QPC. If only a few channels are open the average potential has in the center of the conduction channel the form of a saddle [36]:

$$
V_{e q}(x, y)=V_{0}+\frac{1}{2} m \omega_{y}^{2} y^{2}-\frac{1}{2} m \omega_{x}^{2} x^{2},
$$

where $V_{0}$ is the potential at the saddle and the curvatures of the potential are parametrized by $\omega_{x}$ and $\omega_{y}$. The resulting transmission probabilities have the form of Fermi functions $T(E)=1 /\left(e^{\beta(E-\mu)}+1\right.$ ) (with a negative temperature $\beta=-2 \pi / \hbar \omega_{x}$ and $\left.\mu=\hbar \omega_{y}(n+1 / 2)+V_{0}\right)$. As a function of energy (gate voltage) the conductance rises step-like. The energy derivative of the transmission probability $d T_{n} / d E=\left(2 \pi / \hbar \omega_{x}\right) T_{n}\left(1-T_{n}\right)$ is itself proportional to the transmission probability times the reflection probability. We note that such a relation holds not only for the saddle point model of a QPC but also for instance for the adiabatic model [37. As a consequence $\left(1 / 4 T_{n} R_{n}\right)\left(d T_{n} / d E\right)^{2}=\left(\pi / \hbar \omega_{x}\right)^{2} T_{n} R_{n}$ is proportional to $T_{n} R_{n}$. Thus the charge relaxation resistance of a saddle QPC is

$$
R_{q}=\frac{h}{4 e^{2}} \frac{\sum_{n}\left[\left(\frac{d \phi_{n}}{d E}\right)^{2}+\left(\frac{\pi}{\hbar \omega_{x}}\right)^{2} T_{n} R_{n}\right]}{\left[\sum_{n} \frac{d \phi_{n}}{d E}\right]^{2}} .
$$

To find the density of states of the $n$-th eigen channel, we use the relation 


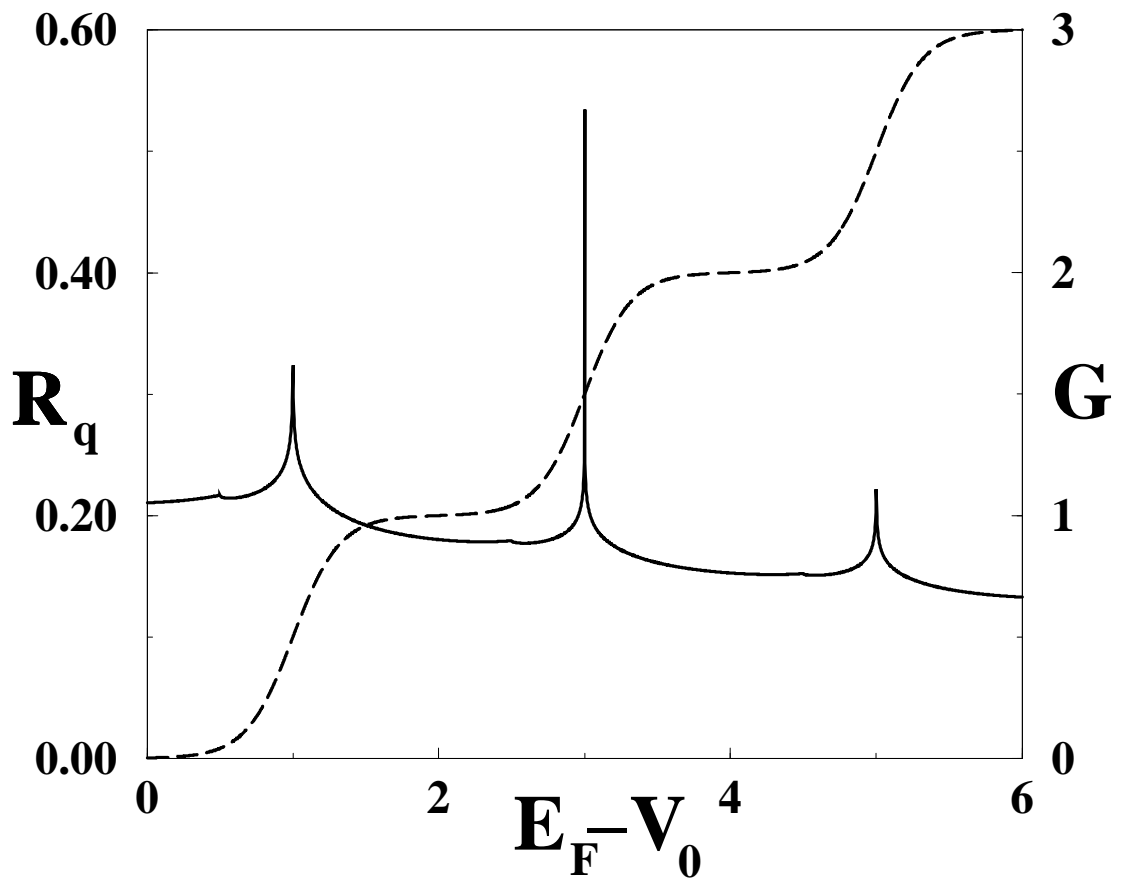

Fig. 2. Charge relaxation resistance $R_{q}$ of a saddle QPC in units of $h / 4 e^{2}$ for $\omega_{y} / \omega_{x}=2$ and a screening length of $m \omega_{x} \lambda^{2} / \hbar=2 E_{\lambda} / \hbar \omega_{x}=25$ as a function of $E_{F}-V_{0}$ in units of $\hbar \omega_{x}$ (full line). The broken line shows the conductance of the QPC. After [10].

between density and phase (action) and $D_{n}=\sum_{i} \mathcal{D}_{n, i i}=(1 / \pi) d \phi_{n} / d E$. We evaluate the phase semi-classically. The spatial region of interest for which we have to find the density of states is the region over which the electron density in the contact is not screened completely. We denote this length by $\lambda$ and the associated energy by $E_{\lambda}=(1 / 2) m \omega_{x}^{2} \lambda^{2}$. The density of states is then found from $D_{n}=1 / h \int_{-\lambda}^{\lambda} \frac{d p_{n}}{d E} d x$ where $p_{n}$ is the classically allowed momentum. A calculation gives a density of states [2] $D_{n}(E)=\left(4 /\left(h \omega_{x}\right)\right) \operatorname{asinh}\left[E_{\lambda} /\left(E-E_{n}\right)\right]^{1 / 2}$, for energies $E$ exceeding the channel threshold $E_{n}$ and gives a density of states $D_{n}(E)=\left(4 /\left(h \omega_{x}\right)\right) \operatorname{acosh}\left[E_{\lambda} /\left(E_{n}-E\right)\right]^{1 / 2}$ for energies in the interval $E_{n}-E_{\lambda}$ $\leq E<E_{n}$ below the channel threshold. Electrons with energies less than $E_{n}-E_{\lambda}$ are reflected before reaching the region of interest, and thus do not contribute to the density of states. The resulting density of states has a logarithmic singularity at the threshold $E_{n}=\hbar \omega_{y}\left(n+\frac{1}{2}\right)+V_{0}$ of the $n$-th quantum channel. (A fully quantum mechanical calculation gives a density of states which exhibits also a peak at the threshold but which is not singular). 
We now have all the elements to calculate the charge relaxation resistance $R_{q}$ and the resistance $R_{v}$. The charge relaxation resistance for a saddle QPC is shown in Fig. 2 for a set of parameters given in the figure caption. The charge relaxation resistance exhibits a sharp spike at each opening of a quantum channel. Physically this implies that the relaxation of charge, determined by the $R C$-time is very rapid at the opening of a quantum channel.

Similarly, we can find the resistance $R_{v}$ for a QPC subject to a voltage $e|V|>>k T$. Using the density matrix elements for a symmetric QPC given by Eqs. (20), we find [2]

$$
R_{v}=\frac{h}{e^{2}} \frac{\sum_{n} \frac{1}{4 R_{n} T_{n}}\left(\frac{d T_{n}}{d E}\right)^{2}}{\left[\sum_{n}\left(d \phi_{n} / d E\right)\right]^{2}}=\frac{h}{e^{2}}\left(\frac{\pi}{\hbar \omega_{x}}\right)^{2} \frac{\sum_{n} T_{n} R_{n}}{\left[\sum_{n}\left(d \phi_{n} / d E\right)\right]^{2}}
$$

The resistance $R_{v}$ is shown in Fig. 3 .

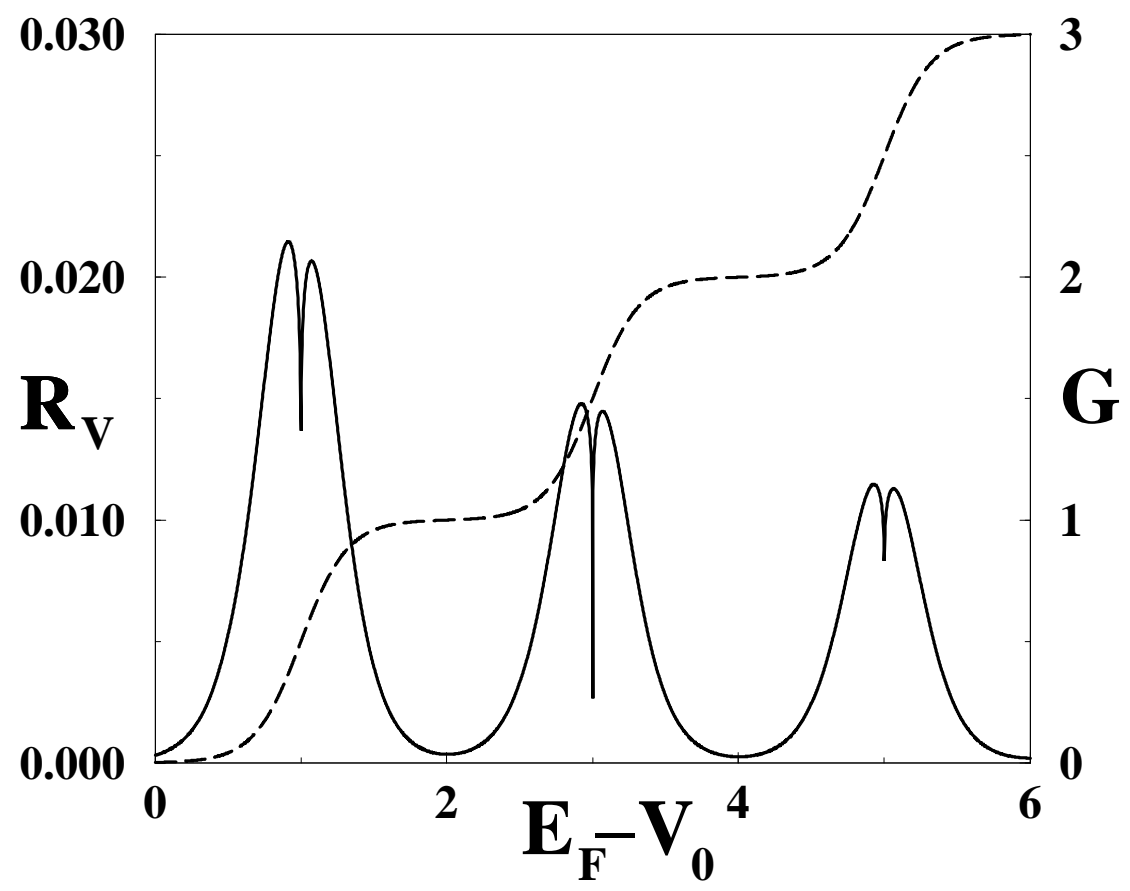

Fig. 3. $R_{v}$ (solid line) for a saddle QPC in units of $h / e^{2}$ and $G$ (dashed line) in units of $e^{2} / h$ as a function of $E_{F}-V_{0}$ in units of $\hbar \omega_{x}$ with $\omega_{y} / \omega_{x}=2$ and a screening length $m \omega_{x} \lambda^{2} / \hbar=25$. $R_{v}$ and $G$ are for spinless electrons. 


\section{Dephasing rates and potential fluctuations}

Let us now use the results discussed above to find the dephasing rates in the Coulomb coupled conductors. Consider a scattering state $\Psi_{i}(\mathbf{r}, E)$ at energy $E$ in conductor $i$ which solves the Schrödinger equation for a fixed potential $U_{e q, i}(\mathbf{r})$. Fluctuations of the potential $U_{i}(\mathbf{r}, t)$ away from the static (average) equilibrium potential will scatter the carrier out of the eigenstate $\Psi_{i}(\mathbf{r}, E)$. Here we regard the fluctuating potential in the interior of the conductor as spatially uniform. Thus the fluctuating potential $d U_{i}(t)=U_{i}(t)-U_{e q, i}$ in the region of interest is a function of time only. The effect of the fluctuating potential can then be described with the help of a time-dependent phase $\phi(t)$ which multiplies the scattering state. Thus we consider a solution of the type $\Psi_{i}(\mathbf{r}, E) \exp (-i \phi(t))$ of the time dependent Schrödinger equation. The equation of motion for the phase is simply, $\hbar d \phi / d t=e d U_{i}(t)$. Let us characterize the potential fluctuations in conductor $i$ by its noise spectrum $S_{U_{i} U_{i}}(\omega)$. Using the noise spectrum $S_{U_{i} U_{i}}(\omega)$ of the voltage fluctuations we find that at long times the phase $\phi$ of the scattering state diffuses with a rate

$$
\Gamma_{\phi}^{(i)}=\left\langle(\phi(t)-\phi(0))^{2}\right\rangle / 2 t=\left(e^{2} / 2 \hbar^{2}\right) S_{U_{i} U_{i}}(0)
$$

determined by the zero-frequency limit of the noise power spectrum of the potential fluctuations.

Eq. (12) shows that the potential fluctuations and thus the dephasing rate has two sources: A carrier in conductor $i$ suffers dephasing due to charge fluctuations in conductor $j=i$ itself, and due to charge fluctuations of the additional nearby conductor $j \neq i$. Accordingly, we can also write the dephasing rate in conductor $i$ as a sum of two contributions, $\Gamma_{\phi}^{(i)}=\sum_{j} \Gamma_{\phi}^{(i j)}$ with

$$
\Gamma_{\phi}^{(i j)}=\left(e^{4} / 2 \hbar^{2}\right) G_{i j}^{2} S_{N_{j} N_{j}}(0)
$$

At equilibrium, we can express the charge noise power with the help of the equilibrium charge relaxation resistances $R_{q}^{j}$ given by Eq. (13). Of particular interest is the dephasing rate in the cavity (conductor $i=0$ ) due to the presence of the QPC (conductor $i=1$ ). The self-consistent theory gives for this dephasing rates at equilibrium 10

$$
\Gamma_{\phi}^{(01)}=\left(e^{2} / \hbar^{2}\right)\left(e^{2} D_{1} G_{01}\right)^{2} R_{q}^{(1)} k T .
$$

In the non-equilibrium case, if conductor 1 is subject to a current generated by a voltage $|V|$ (with $k T<<e|V|)$ we find a dephasing rate [10]

$$
\Gamma_{\phi}^{(01)}=\left(e^{2} / \hbar^{2}\right)\left(e^{2} D_{1} G_{01}\right)^{2} R_{v}^{(1)} e|V| .
$$

These dephasing rates are proportional to $\left(e^{2} D_{1} G_{01}\right)^{2}$. Thus $e^{2} D_{1} G_{01}$ plays the role of an effective coupling constant in our problem. In the limit of a small Coulomb energy $\left(C \gg e^{2} D_{0}\right.$ and $\left.C \gg e^{2} D_{1}\right)$ we find $e^{2} D_{1} G_{01}=D_{0} /\left(D_{0}+D_{1}\right)$ independent of the geometrical capacitance $C$. In the limit of a large Coulomb 
energy ( $C \ll e^{2} D_{0}$ and $C \ll e^{2} D_{1}$ ) the effective coupling constant becomes proportional to the capacitance $e^{2} D_{1} G_{01}=C /\left(e^{2} D_{0}\right)$. The second limit, typically, is the physically relevant limit.

According to Eq. (27) and Eq. (28), in an experiment in which the QPC is opened with the help of a gate, the dephasing rate follows, at equilibrium just $R_{q}$ and in the non-equilibrium case follows $R_{v}$. Without screening $R_{v}$ would exhibit a bell shaped behavior as a function of energy, i. e. it would be proportional to $T_{n}\left(1-T_{n}\right)$ in the energy range in which the $\mathrm{n}$-th transmission channel is partially open. Screening, which in $R_{v}$ is inversely proportional to the density of states squared, generates the dip at the threshold of the new quantum channel at the energy which corresponds to $T_{n}=1 / 2$ (see Fig. 3). It is interesting to note that the experiment [3] does indeed show a double hump behavior of the dephasing rate.

\section{Self-consistent measurement time}

Suppose that a measurement of the current is used to determine the charging state of the cavity. Consider the two charging states with $Q_{0}$ and $Q_{0}+e$ electrons on the cavity. The two charge states on the cavity give, via long range Coulomb interaction, rise to a conductance $G\left(\right.$ charge $\left.Q_{0}\right)$ and $G+\Delta G$ (charge $Q_{0}+e$ ). The measurement time $\tau_{m}$ is the (minimal) time needed to determine the conductance through a measurement that flows through the QPC. A measurement needs to overcome the fluctuations of the current (shot noise and thermal noise). The measurements needs to be long enough [月, 3 so that the integrated current $\Delta G|V| \tau_{m}$ due to the variation of the conductance, exceeds the integrated current fluctuation $\sqrt{S_{I I}(0) \tau_{m}}$. Here, $S_{I I}(0)$ is the low frequency spectral current density, which in the zero temperature limit is due to shot nose alone and given by [34.35.11 $S_{I I}(0)=2 e\left(e^{2} / h\right)|V| \sum_{n} T_{n} R_{n}$. This gives a measurement time

$$
\tau_{m}=\frac{S_{I I}}{(\Delta G)^{2}|V|^{2}} \text {. }
$$

$\Delta G$ is another quantity that can be measured independently and such a measurement has in fact been carried out by Buks et al. [3]. It is thus useful to make a theoretical prediction for this quantity. Therefore our purpose here is to evaluate $\Delta G$ and compare the expression for the measurement time with the phase breaking rate obtained above.

The variation of the conductance of the QPC is determined by the sensitivity of the conductance due to the variation of the potential $d U_{1}$ in the QPC

$$
\Delta G=\left(e^{2} / h\right)\left(d T / d U_{1}\right) d U_{1} .
$$

Here $T$ is the total transmission probability, $T \equiv \sum_{n} T_{n}$. In Eq. (30) $U_{1}$ is the potential in the QPC and $d U_{1}$ is the change in potential for the case that an additional electron eners the cavity. In WKB-approximation we are allowed to replace the derivative with respect to the potential with a derivative with 
respect to energy, $d T / d U_{1}=-e d T / d E$. For the saddle point QPC we have $d T / d E=\left(2 \pi / \hbar \omega_{x}\right) \sum_{n} T_{n} R_{n}$. Form Eq. (5) we find with $e d N_{0}=e$, a potential variation $d U_{1}=G_{10} e d N_{0}=e G_{10}$ where $G_{10}$ is an off-diagonal element of the effective interaction matrix, Eq. (7). Thus the addition of an electron onto the cavity changes the conductance by [38]

$$
\Delta G=-\left(e^{2} / h\right) e G_{10} d T / d E .
$$

Since $G_{10}=C_{\mu} /\left(e^{2} D_{0} e^{2} D_{1}\right)$ we find in the limit $e^{2} / C>>1 / D_{1}, e^{2} / C>>$ $1 / D_{2}$,

$$
\Delta G=-\left(e^{2} / h\right)\left(C / e^{2} D_{0}\right)\left(e(d T / d E) / e^{2} D_{1}\right) .
$$

As discussed above, as a function of gate voltage the density of states $D_{1}$ of the QPC exhibits a strong variation. In particular, at zero temperature, the density of states diverges at the threshold of a new quantum channel. Consequently, $\Delta G$ is also a strong function of gate voltage. $\Delta G$ vanishes on the conductance plateaus since $(d T / d E)$ vanishes on a plateau. Eq. (31) predicts that $\Delta G$ vanishes also at the channel opening threshold $\left(T_{n}=1 / 2\right)$ since the semiclassical density of states diverges. Thus Eq. (31) predicts that $\Delta G$ (like $R_{v}$ ) is maximal away from the channel opening threshold. Such a behavior is not seen in the experiment of Buks et al. [3], $\Delta G$ seems to be rather independent of the gate voltage. This can be due to the simple model of the QPC used here or due to the fact that the experiment is not in the zero-temperature limit.

We now return to the measurement time, Eq. (29). Using Eq. (31) and $d T / d E=\left(2 \pi / \hbar \omega_{x}\right) \sum_{n} T_{n} R_{n}$, and the expression for $R_{v}$ as given by Eq. (24) we find,

$$
\tau_{m}=\frac{2 e^{2}}{\pi^{2}\left(e^{2} / h\right)^{2}\left[e^{2} G_{10} D_{1}\right]^{2} R_{v} e|V|} .
$$

Comparison with our result for $\Gamma_{\phi}$ (see Eq. (28)) shows that $(1 / 2) \Gamma_{\phi} \tau_{m}=1$ which agress with Korotkov [17]. As mentioned in the introduction, the identification of the measurement time $\tau_{m}$ with $1 / \Gamma_{\phi}$ is taken for granted by a several authors. It is now, however, clear that in general [13.17] $(1 / 2) \Gamma_{\phi} \tau_{m}>1$ ( for instance for a detector that is not symmetric). Even for the symmetric detector (symmetric QPC) considered here, it is clear that a non-zero temperature has a different effect on the dephasing time and on the measurement time. The dephasing time $\tau_{\phi}=1 / \Gamma_{\phi}$ is inversely proportional to the charge fluctuation spectrum whereas the measurement time is proportional to the current fluctuation spectrum. At elevated temperatures, the measurement must overcome the combined thermal and shot noise and the measurement time will thus increase with increasing temperature. On the other hand the additional Nyquist noise leads to a shorter dephasing time.

\section{Discussion}

We have presented an electrically self-consistent discussion of dephasing rates and measurement times for Coulomb coupled conductors. The approach empha- 
sizes that also in open conductors, like QPC's, charge fluctuations are associated with a Coulomb energy. In such a self-consistent treatment, the dephasing rates are typically not simply proportional to a coupling constant. In this work, we have attributed only a single potential to each conductor, but the theory [10] is not in fact limited to such a simplification and permits the treatment of an arbitrary potential landscape $\sqrt{10,39}$. The theory also permits a discussion of a wide variety of geometries 39.27 .

We have treated the charge fluctuations within a linear screening approach. Large changes in the potential of the QPC would require a discussion of nonlinear screening. In either case, a theory is necessary which treats the true charge distribution and its fluctuations. A carrier on the cavity is entangled not only with a single electron on the QPC but with all electrons which are involved in the screening process on the cavity and on the QPC. Instead of a few electron problem our approach emphasizes the true many body nature of charge fluctuations of Coulomb coupled conductors. We have restricted our considerations to the case where we deal with open conductors for which charge quantization plays a minor role. The considerations given above apply, however, also to the case where we have a QPC interacting with a system in which charge is quantized. Even in this case carriers on the QPC will be screened to a certain extent and the charge relaxation resistance $R_{q}$ and the resistance $R_{v}$ should again be part of a self-consistent answer.

\section{Acknowledgement}

This work was supported by the Swiss National Science Foundation and the TMR network.

\section{References}

1. Ya. M. Blanter and M. Büttiker, Physics Reports, 336, 1-166 (2000).

2. M. H. Pedersen, S. A. van Langen and M. Büttiker, Phys. Rev. B 57, 1838 (1998).

3. E. Buks, R. Schuster, M. Heiblum, D. Mahalu and V. Umansky, Nature 391, 871 (1998).

4. D. Sprinzak, E. Buks, M. Heiblum and H. Shtrikman, Phys. Rev. Lett. 84, 5820 (2000).

5. R. A. Harris and L. Stodolsky, J. Chem. Phys. 74, 2145 (1981); Phys. Lett. B116, 464 (1982).

6. S. A. Gurvitz, Phys. Rev. B 56, 15215 (1997).

7. I. L. Aleiner, N. S. Wingreen, and Y. Meir, Phys. Rev. Lett. 79, 3740 (1997).

8. Y. B. Levinson, Europhys. Lett. 39, 299 (1997).

9. L. Stodolsky, Phys. Lett. B 459, 193 (1999).

10. M. Büttiker and A. M. Martin, Phys. Rev. B61, 2737 (2000).

11. M. Büttiker, in Statistical and Dynamical Aspects of Mesoscopic Systems, edited by D. Reguera, G. Platero, L. L. Bonilla and J. M. Rubi, (Springer, Berlin, 2000). Lecture Notes in Physics, Vol. 547, p. 81.

12. Y. B. Levinson, Phys. Rev. B 61, 4748 (2000). 
13. A. Shnirman and G. Schön, Phys. Rev. B57, 15400 (1998).

14. Y. Makhlin, G. Schön, A. Shnirman, (unpublished). cond-mat/0001423

15. A. N. Korotkov, D. V. Averin, (unpublished). cond-mat/0002203

16. D.V. Averin. In Exploring the Quantum-Classical Frontier: Recent Advances in Macroscopic and Mesoscopic Quantum Phenomena, Eds. J.R. Friedman and S. Han, (unpublished). cond-mat/0004364

17. A. N. Korotkov, cond-mat/0008461

18. T. Christen and M. Büttiker, Phys. Rev. Lett. 77, 143 (1996)

19. J. Wang, H. Guo, J.-L. Mozos, C. C. Wan, G. Taraschi, and Q. Zheng, Phys. Rev. Lett. 80, 4277 (1998).

20. B. G. Wang, X. Zhao, J. Wang, et al. Appl. Phys. Lett. 74, 2887 (1999).

21. M. Büttiker and T. Christen, in Mesoscopic Electron Transport, NATO Advanced Study Institute, Series E: Applied Science, edited by L. L. Sohn, L. P. Kouwenhoven and G. Schoen, (Kluwer Academic Publishers, Dordrecht, 1997). Vol. 345. p. 259.

22. M. Büttiker, H. Thomas, and A. Prêtre, Phys. Lett. A 180, 364 (1993).

23. M. Büttiker, J. Math. Phys., 37, 4793 (1996).

24. T. Christen and M. Büttiker, Europhys. Lett. 35, 523 (1996).

25. Z.-s. Ma, J. Wang and H. Guo, Phys. Rev. B 57, 9108 (1988).

26. E. G. Emberly, G. Kirczenow, cond-mat/0009386

27. M. Büttiker, in "Quantum Mesoscopic Phenomena and Mesoscopic Devices", edited by I. O. Kulik and R. Ellialtioglu, (Kluwer, unpublished). cond-mat/9911188

28. The Lindhard function is $\pi\left(\mathbf{r}, \mathbf{r}^{\prime}\right) \equiv \partial \rho(\mathbf{r}) / \partial U\left(\mathbf{r}^{\prime}\right)$ with $\rho$ the local density and $U$ the local potential. Since we assume a single spatially uniform potential and since we are interested in the total charge on the conductor in the volume (area) $\Omega$ we have $\int d^{3} \mathbf{r} \int d^{3} \mathbf{r}^{\prime} \pi\left(\mathbf{r}, \mathbf{r}^{\prime}\right)=-e^{2} \int d^{3} \mathbf{r} \nu(\mathbf{r})=-e^{2} D$. Here $\nu(\mathbf{r})$ is the local density of states and $D$ denotes the total integrated density of states of the conductor.

29. V. Gasparian, T. Christen, and M. Büttiker, Phys. Rev. A 54, 4022 (1996).

30. X. Zhao, J. Phys. Cond. Matter, 12, 4053 (2000).

31. Ya. M. Blanter, F.W.J. Hekking, and M. Büttiker, Phys. Rev. Lett. 81, 1925 (1998).

32. P. W. Brouwer and M. Büttiker, Europhys. Lett. 37, 441-446 (1997).

33. F. T. Smith, Phys. Rev. 118349 (1960).

34. G. B. Lesovik, JETP Lett. 49, 592 (1989).

35. M. Büttiker, Phys. Rev. Lett. 65, 2901 (1990); Phys. Rev. B 46, 12485 (1992).

36. M. Büttiker, Phys. Rev. B 41, 7906 (1990).

37. L. I. Glazman, G. B. Lesovik, D. E. Khmel'nitskii, and R. I. Shekhter, JETP Lett. 48, 238 (1988).

38. For an ensemble of chaotic cavities the distribution of this conductance derivative is given by P. W. Brouwer, S. A. van Langen, K. M. Frahm, M. Büttiker, and C. W. J. Beenakker, Phys. Rev. Lett. 79, 914 (1997).

39. A. M. Martin and M. Büttiker, Phys. Rev. Lett. 84, 3386 (2000). 